\title{
Clinodactyly and syndactyly - diagnostic clues for Andersen-Tawil syndrome
} Clinodactilia e sindactilia - pistas diagnósticas da síndrome de Andersen-Tawil Carlos Andrade ${ }^{1,4}$, Joana Meireles ${ }^{1,4}$, Miguel Leão ${ }^{2,3}$, Fernando Silveira ${ }^{1}$

A 38-year-old man was diagnosed, at the age of 18, with SCN4A-negative hyperkalaemic periodic paralysis. The diagnosis remained unchanged until his 8-year-old daughter suffered an exercise-induced syncope. Her EKG showed a polymorphic ventricular tachycardia. Patient's hands and feet, previously overlooked, became "neurologically" relevant since they were characteristic of Andersen-Tawil syndrome (Figure). A pathogenic KCNJ2 mutation (Arg218Trp) was found.

Andersen-Tawil syndrome is an autosomal dominant disorder characterized by the triad of periodic paralysis, ventricular arrhythmias, and dysmorphic features ${ }^{1}$. Phenotypical heterogeneity, even within a family, often delays the diagnose which is necessary since cardiac assessment is warrant ${ }^{2}$.
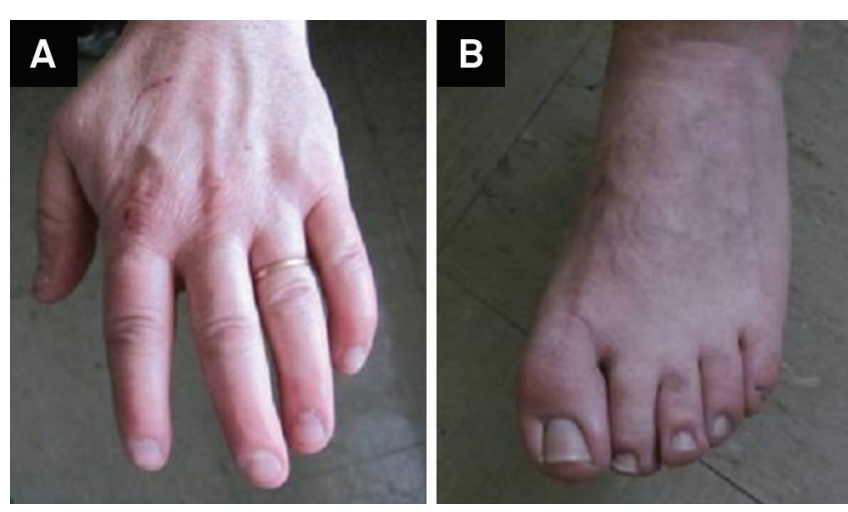

Figure. (A) Fifth digit clinodactyly and (B) syndactyly of the toes 2 and 3 , highly suggestive of Andersen-Tawil syndrome. The face (not shown) had only mild phenotypical characteristics.

\section{References}

1. Tawil R, Ptacek LJ, Pavlakis SG, DeVivo DC, Penn AS, Ozdemir C et al. Andersen's syndrome: potassium-sensitive periodic paralysis, ventricular ectopy, and dysmorphic features. Ann Neurol.1994;35(3):326-30. http://dx.doi.org/10.1002/ana.410350313
Davies NP, Imbrici P, Fialho D, Herd C, Bilsland LG, Weber A, et al. Andersen-Tawil syndrome: new potassium channel mutations and possible phenotypic variation. Neurology. 2005;65:1083-9.

'Departamento de Neurologia, Centro Hospitalar de São João, Porto, Portugal;

${ }^{2}$ Unidade de Neurologia Pediátrica, Departamento de Pediatria, Centro Hospitalar de São João, Porto, Portugal;

${ }^{3}$ Departamento de Genetica Médica, Centro Hospitalar de São João, Porto, Portugal;

${ }^{4}$ Departamento de Neurociências Clínicas e Saúde Mental, Universidade do Porto, Porto, Portugal.

Correspondence: Carlos Jorge da Silva Andrade; Departamento de Neurologia, Centro Hospitalar de São João; Alameda Prof. Hernâni Monteiro, 4200 / 319 Porto, Portugal; E-mail: carlos.hsjn@gmail.com

Conflict of interest: There is no conflict of interest to declare.

Received 14 June 2014; Received in final form 31 July 2014; Accepted 19 August 2014. 